\title{
139. An analytical modeling for high-velocity impacts on woven Kevlar composite laminates
}

\author{
Chen Fangyu ${ }^{1}$, Zhou $\mathrm{Li}^{2}$, Tang Yihao ${ }^{3}$, Zhou Xiaoming ${ }^{4}$ \\ ${ }^{1,2}$ State Key Laboratory of Mechanics and Control of Mechanical Structures, \\ Nanjing University of Aeronautics and Astronautics, Nanjing, China \\ ${ }^{3,4}$ China Helicopter Research and Development Institute, Jingdezhen, China \\ ${ }^{2}$ Corresponding author \\ E-mail: ${ }^{1}$ cfy18405008@avic.com, ${ }^{2}$ lzhou@nuaa.edu.cn, ${ }^{3}$ tyh3786@163.com, ${ }^{4670318048 @ q q . c o m ~}$
}

Received 8 October 2017; accepted 27 October 2017

DOI https://doi.org/10.21595/jme.2017.19253

Check for updates

\begin{abstract}
In this paper, an analytical model, which based on energy balance, is built to study the process of high velocity impacts on woven Kevlar composite laminates by a cylindrical projectile. Four different mechanisms, such as laminate crushing, linear momentum transfer and tensile fiber failure, and shear plugging, is absorbed by the laminate while impacting. Then, simplification of the model is done to obtain the residual velocity and ballistic limit. The analytical results are validated with the results of experiment, and the perturbation analysis is done to analyze the reason of error.
\end{abstract}

Keywords: high velocity impacts, composite laminates, residual velocity, ballistic limit.

\section{Introduction}

Resistance to ballistic impacts is an important requirement in many industrial applications, such as car, space shuttle and aeronautical industry. Woven fabrics are well-known to be good choice to resist ballistic impacts [1-3]. In the past, several researches have been focused on the impact on composite laminates. Elasticity method [4-7] was widely used to predict the behavior of impact. Three phases, which namely indentation, perforation and exit, are divided in the impact event by Zhu et al. [4]. Sun et al. [5] applied different criteria to obtain damage initiation, progression and plug formation. Wen et al. $[6,7]$ applied projectiles with different shapes to investigate FRP laminates' perforation and penetration. Another method to predict the behavior of impact was the energy method. The projectile's residual velocity and ballistic limit are calculated by energy balance equation. Naik et al. [8] and Xuan Haijun et al. [9] study the perforation of the composite plates with all the damage mechanisms and verified the analytical results with experimental results. Impact test is done by Ganesh Babu et al. [10] using different shape projectiles on unidirectional glass/epoxy composite plates. Sikarwar et al. [11, 12] and Wang Yuanbo et al. $[13,14]$ studied the analytical and experimental results of the residual velocity and ballistic limit of glass/epoxy and Kevlar/epoxy composite laminates respectively, which are subjected to high velocity impact. Yahaya et al. [15] observed a linear increasing tendency of energy absorption against impact velocity for Kevlar-Kenaf hybrid woven composites. Gu Xingjin et al. [16] and Michele et al. [17] calculate energy absorbed in different mechanisms with flat-end cylindrical projectiles. Chen et al. [18] studied simulation and experimental results of damage and energy absorption behavior for woven composites under ballistic impact. The researches mentioned above spend a lot of attention to high precision of the solutions of different equations, so they are high cost. To make a quick assessment at early stages of the design process, Lopez-Puente et al. neglect elastic deformation contribution in impact event [19] and then proposes a low-cost model and obtains closed-form solution to predict the ball projectile's residual velocity after impacting on thin CFRPs woven laminated plates [20], but the influence of shear plugging [21-23] is not considered in their research.

In this paper, an analytical model is proposed to build the energy balance equation and predict the residual velocity of a cylindrical projectile after impacting at woven Kevlar composites. Simplification of the model is done to obtain the residual velocity and ballistic limit. Also, three 
dimensionless variables are built to simplify the process of analysis perturbation, which caused by tensile fiber failure. Analysis is done to explain the error between the results of calculation and experiment [13]. The model is low-cost, and its predictive accuracy is improved. The method of simplification could be useful in preliminary assessment of engineering.

\section{Analytical model}

The analytical model is built by terms of energy balance. In Lopez-Puente et al.' [17] opinion, the kinetic energy of the projectile $E_{k}$ is absorbed by the laminate through three different processes. $E_{c}$ is the energy of crushing, which leads to laminate breakage. $E_{m}$ is the energy of linear momentum, transferred from the projectile to the detached part of laminate. $E_{f}$ is the energy of tensile fiber failure breakage. Elastic deformation energy is not considered by them in this analytical model. Naik et al. [19, 20] and Brenda et al. [21] consider that $E_{s p}$, the energy absorbed by shear plugging, is also an important process. So, the basic balance equation can be presented in the following because of the influence of the shear plugging:

$-d E_{k}=d E_{c}+E_{m}+d E_{f}+d E_{s p}$.

Following each energetic term of Eq. (1) is presented.

The kinetic energy of the projectile is expressed as:

$d E_{k}(x)=\frac{1}{2} m_{p} d\left(v(x)^{2}\right)$,

where $x$ is the distance between the position of the projectile and the impact surface of the composite laminate, $m_{p}$ is the mass of projectile, and $v$ is the velocity varies with the position.

The energy of laminate crushing is expressed as:

$d E_{c}(x)=\sigma_{c} A(x) d x$,

where $A(x)$ is the frontal projectile area that contacts the non-crushed laminate and $\sigma_{c}$ is the out-of-plane compressive strength of the laminate. Here, $A(x)=\pi r^{2}$, where $r$ denotes the radius of the projectile cross-section.

The energy of linear momentum transfer is expressed as:

$d E_{m}(x)=\frac{1}{2}(A(x) d x \rho) v^{2}(x)$,

where $\rho$ is the density of laminate.

The energy of tensile fiber failure is expressed as:

$d E_{f}(x)=\omega_{f} d V$

where $\omega_{f}$ is the specific energy and $d V$ is the affected material volume at any $d x$. The specific energy is estimated as:

$\omega_{f}=2\left(\frac{1}{2} X_{t} \varepsilon_{f}\right)$

where $X_{t}$ is the tensile strength in fiber direction and $\varepsilon_{f}$ is the ultimate strain. Experimental result $[9,20]$ shows that the transverse section of laminate is a truncated pyramid, with a semi-angle $\alpha$ and $\beta$, and its diagonals aligned in the fiber directions. The upper base semi-diagonal is $r$ and the 
lower base semi-diagonal is $L=r+\left(h-x_{0}\right) \tan \alpha$ with $x_{0}$ the position of the projectile at the time $x_{0}$, as Fig. 1 shown. The affected material volume $d V$ is given by:

$d V=2 l \times l_{w} d x=2 d x\left(r+\left(h-x_{0}\right) \tan \alpha\right)^{2} \tan \beta$,

and the energy of tensile fiber failure is given by:

$d E_{f}(x)=2 X_{t} \varepsilon_{f}\left(r+\left(h-x_{0}\right) \tan \alpha\right)^{2} \tan \beta d x$.

The value of $x_{0}$ can be obtained from following equation:

$\int_{0}^{x_{0}} \frac{d x}{v(x)}=\frac{2 h}{\sqrt{E_{3} / \rho}}$

where $h$ is the thickness of laminate and $E_{3}$ is the elastic modulus through-thickness.

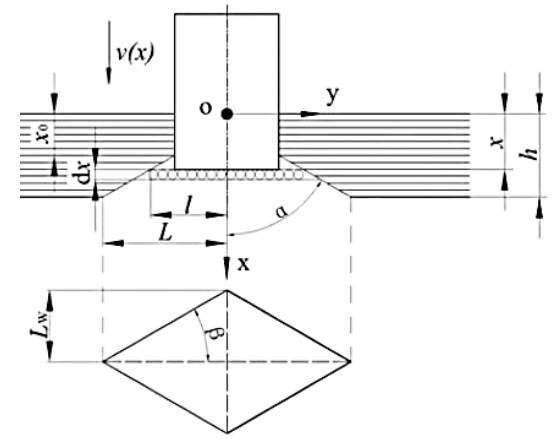

Fig. 1. Woven composite with cylindrical projectile

Tensile fiber failure happens while $x_{0}<x<h$, so a function $C_{r}(x)$ is defined as follow to multiplies the last equation:

$C_{r}(x)= \begin{cases}0, & 0<x<x_{0} \\ 1, & x_{0}<x<h \\ 0, & x \geq h\end{cases}$

The energy absorbed of shear plugging is expressed as:

$d E_{s p}(x)=S_{s p} S(x) x d x$

where $S_{s p}$ is shear plugging strength and $S(x)$ is the cross-section perimeter of the projectile. Here, $S(x)=2 \pi r$.

While $x \geq x_{0}$, the crushing energy and shear plugging energy have no influence. So, a function $C_{s}(x)$ is defined as follow to multiply the Eq. (3) and Eq. (4):

$C_{s}(x)= \begin{cases}1, & 0<x<x_{0} \\ 0, & x \geq x_{0}\end{cases}$

\section{Model simplification}

As analysis above, the basic balance equation can be written as: 


$$
\begin{aligned}
- & \frac{1}{2} m_{p} d\left(v(x)^{2}\right)=\sigma_{c} A(x) C_{s}(x) d x+\frac{1}{2}(A(x) d x \rho) v^{2}(x) \\
& +2 X_{t} \varepsilon_{f}\left(r+\left(h-x_{0}\right) \tan \alpha\right)^{2} \tan \beta C_{r}(x) d x+S_{s p} S(x) x C_{s}(x) d x .
\end{aligned}
$$

To solve the equation, some simplifications are made and the initial condition is imported:

$$
\left\{\begin{array}{l}
-\frac{1}{2} m_{p} \frac{d^{2} v}{d x}=\frac{1}{2}(A(x) \rho) v^{2}+S_{s p} S(x) x C_{s}(x)+\sigma_{c} A(x) C_{s}(x) \\
\quad+2 X_{t} \varepsilon_{f}\left(r+\left(h-x_{0}\right) \tan \alpha\right)^{2} \tan \beta C_{r}(x), \\
v(0)=v_{0} .
\end{array}\right.
$$

Here the velocity and the displacement are converted to dimensionless variables:

$$
v^{*}=\frac{v}{v_{0}}, x^{*}=\frac{x}{h} \text {. }
$$

By defining a variable $y^{*}=v^{*^{2}}$, Eq. (14) leads to:

$$
\left\{\begin{array}{l}
-\frac{d y^{*}}{d x^{*}}=\frac{\pi r^{2} h \rho}{m_{p}} y^{*}+\frac{4 S_{s p} \pi r h^{2}}{m_{p} v_{0}^{2}} C_{s}\left(h x^{*}\right) x^{*}+\frac{2 \pi r^{2} h \sigma_{c}}{m_{p} v_{0}^{2}} C_{s}\left(h x^{*}\right) \\
+\frac{4 X_{t} \varepsilon_{f} r^{2} h}{m_{p} v_{0}^{2}}\left(1+\left(\frac{h}{r} x^{*}-\frac{x_{0}}{r}\right) \tan \alpha\right)^{2} \tan \beta C_{r}\left(h x^{*}\right), \\
y^{*}(0)=1 .
\end{array}\right.
$$

To simplify the process of finding solution, the relative importance of every energy absorption mechanisms are estimated firstly. Then, inconsequential factors can be found and ignored.

The characteristic values of different variables involved are showed in Table 1.

Some variables are defined as a function of the impact velocity to estimate the relative importance of the terms that appear in the equation:

$$
\left\{\begin{array}{l}
R_{m}=\frac{\pi r^{2} h \rho}{m_{p}}-10^{-2}, \\
R_{s p}=\frac{4 S_{s p} \pi r h^{2}}{m_{p} v_{0}^{2}}-\frac{10^{3}}{v_{0}^{2}}, \\
R_{c}=\frac{2 \pi r^{2} h \sigma_{c}}{m_{p} v_{0}^{2}}-\frac{10^{3}}{v_{0}^{2}}, \\
R_{f}=\frac{4 X_{t} \varepsilon_{f} r^{2} h}{m_{p} v_{0}^{2}}-\frac{10}{v_{0}^{2}} .
\end{array}\right.
$$

Obviously, the value of $R_{f}$ is very small. Within the velocity range considered, $R_{f}$ is always lower than the others. The energy of tensile fiber failure is ignored to simplify the model as following:

$$
\left\{\begin{array}{l}
-\frac{d y^{*}}{d x^{*}}=\frac{\pi r^{2} h \rho}{m_{p}} y^{*}+\frac{4 S_{s p} \pi r h^{2}}{m_{p} v_{0}^{2}} C_{s}\left(h x^{*}\right) x^{*}+\frac{2 \pi r^{2} h \sigma_{c}}{m_{p} v_{0}^{2}} C_{s}\left(h x^{*}\right), \\
y^{*}(0)=1 .
\end{array}\right.
$$

The equation can be numerically solved to find the solution: 
$y^{*}=\left\{\begin{array}{l}-\frac{4 S_{s p} h}{v_{0}^{2} r \rho} x^{*}+\frac{4 S_{s p} m_{p}}{v_{0}^{2} \pi r^{3} \rho^{2}}-\frac{2 \sigma_{c}}{v_{0}^{2} \rho}+e^{-\frac{\pi r^{2} h \rho}{m_{p}} x^{*}}\left(-\frac{4 S_{s p} m_{p}}{v_{0}^{2} \pi r^{3} \rho^{2}}+\frac{2 \sigma_{c}}{v_{0}^{2} \rho}+1\right), \quad 0<x^{*}<x_{0}^{*}, \\ -\frac{4 S_{s p} h}{v_{0}^{2} r \rho} x_{0}^{*}+\frac{4 S_{s p} m_{p}}{v_{0}^{2} \pi r^{3} \rho^{2}}-\frac{2 \sigma_{c}}{v_{0}^{2} \rho}+e^{-\frac{\pi r^{2} h \rho}{m_{p}} x_{0}^{*}}\left(-\frac{4 S_{s p} m_{p}}{v_{0}^{2} \pi r^{3} \rho^{2}}+\frac{2 \sigma_{c}}{v_{0}^{2} \rho}+1\right), \quad x^{*} \geq x_{0}^{*} .\end{array}\right.$

$x_{0}^{*}$ can be obtained from following equation:

$\int_{0}^{x_{0}^{*}} \frac{v_{0}}{\sqrt{y^{*}\left(x^{*}\right)}} d x^{*}=\frac{2 h}{\sqrt{E_{3} / \rho}}$

The expression for residual velocity is obtained, which is given by:

$v_{r}=\lim _{x^{*} \rightarrow \infty} v\left(x^{*}\right)=\sqrt{-\frac{4 S_{s p} h}{r \rho} x_{0}^{*}+\frac{4 S_{s p} m_{p}}{\pi r^{3} \rho^{2}}-\frac{2 \sigma_{c}}{\rho}+e^{-\frac{\pi r^{2} h \rho}{m_{p}} x_{0}^{*}}\left(-\frac{4 S_{s p} m_{p}}{\pi r^{3} \rho^{2}}+\frac{2 \sigma_{c}}{\rho}+v_{0}^{2}\right)}$.

For ballistic limit $v_{50}, v_{r}=0$, so ballistic limit $v_{50}$ can be expressed by:

$$
v_{50}=\sqrt{\frac{\frac{4 S_{s p} h}{r \rho} x_{0}^{*}-\frac{4 S_{s p} m_{p}}{\pi r^{3} \rho^{2}}+\frac{2 \sigma_{c}}{\rho}-e^{-\frac{\pi r^{2} h \rho}{m_{p}} x_{0}^{*}}\left(-\frac{4 S_{s p} m_{p}}{\pi r^{3} \rho^{2}}+\frac{2 \sigma_{c}}{\rho}\right)}{e^{-\frac{\pi r^{2} h \rho}{m_{p}} x_{0}^{*}}}} .
$$

Table 1. Characteristic values of different variables

\begin{tabular}{|c|c|}
\hline Variable & Characteristic values \\
\hline$h[\mathrm{~m}]$ & $10^{-3}$ \\
\hline$r[\mathrm{~m}]$ & $10^{-3}$ \\
\hline$m_{p}[\mathrm{~kg}]$ & $10^{-3}$ \\
\hline$\rho\left[\mathrm{kg} / \mathrm{m}^{3}\right]$ & $10^{3}$ \\
\hline$X_{t}\left[\mathrm{~N} / \mathrm{m}^{2}\right]$ & $10^{9}$ \\
\hline$\sigma_{c}\left[\mathrm{~N} / \mathrm{m}^{2}\right]$ & $10^{8}$ \\
\hline$S_{s p}\left[\mathrm{~N} / \mathrm{m}^{2}\right]$ & $10^{8}$ \\
\hline$\varepsilon_{f}$ & $10^{-2}$ \\
\hline
\end{tabular}

\section{Model validation and discussion}

To validate the analytical model above, analytical results are calculated to compare with experimental result [13]. Here cylindrical projectiles with a diameter of $7.62 \mathrm{~mm}$ and a mass of $5.2 \mathrm{~g}$ are used to impact $0^{\circ} / 90^{\circ}$ layup Kevlar laminates with thickness of $5 \mathrm{~mm}$ and $10 \mathrm{~mm}$ respectively. The mechanical properties of Kevlar composite are shown in Table 2.

The projectile residual velocities obtained from different laminate thickness values and projectile initial velocities are shown as Fig. 2. It is clear that the differences between results of calculation and experiment reduce as the initial velocity increases for laminates. For laminate with thickness of $5 \mathrm{~mm}$, the error is about $13 \%$ while the initial velocity below $400 \mathrm{~m} / \mathrm{s}$, and the error is less than $9 \%$ while the initial velocity over $400 \mathrm{~m} / \mathrm{s}$. It shows that the calculated result is closer to experimental result for laminates with thickness of $10 \mathrm{~mm}$ than that of $5 \mathrm{~mm}$. Also, good result was found in the residual velocity estimated by the mentioned method [19], but its error is larger than that found by the proposed model.

The ballistic limit obtained for different laminate thickness values are shown as Fig. 3.

It is clear that the difference between calculated result and experimental result is larger while 
the laminates with thickness of $5 \mathrm{~mm}$ than which of $10 \mathrm{~mm}$. The ballistic limit of calculated result [19] is lower than that of proposed model.

Table 2. Mechanical properties of Kevlar composites

\begin{tabular}{|c|c|}
\hline Mechanical property & Value \\
\hline$\rho\left[\mathrm{kg} / \mathrm{m}^{3}\right]$ & 1440 \\
\hline Modulus in $x$ direction $E_{x}[\mathrm{GPa}]$ & 21 \\
\hline Modulus in $y$ direction $E_{y}[\mathrm{GPa}]$ & 21 \\
\hline Modulus in $z$ direction $E_{z}[\mathrm{GPa}]$ & 4.6 \\
\hline Shear modulus in $x y$ direction $G_{x y}[\mathrm{GPa}]$ & 5.2 \\
\hline$X_{t}[\mathrm{MPa}]$ & 800 \\
\hline$S_{s p}[\mathrm{MPa}]$ & 183 \\
\hline$\sigma_{c}[\mathrm{MPa}]$ & 80 \\
\hline$\varepsilon_{f}$ & 0.02 \\
\hline
\end{tabular}

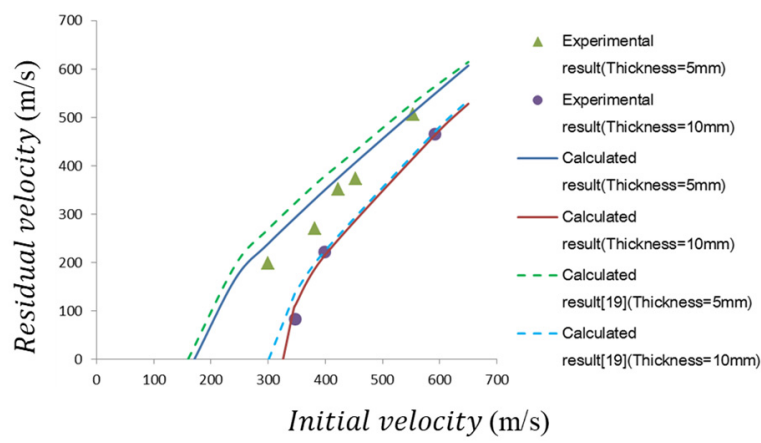

Fig. 2. Comparison of residual velocities obtained from analysis and experiment for different projectile initial velocities

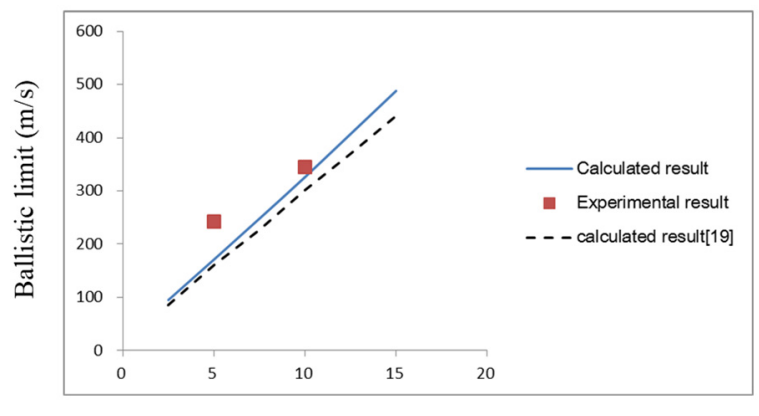

Thickness (mm)

Fig. 3. Comparison of ballistic limit obtained from analysis and experiment for different laminate thickness

The key of explaining the error is analyzing the perturbation caused by tensile fiber failure. It is also important to analyze the influence of the energy absorbed by other three different mechanisms. To simplify the process of analysis and estimate the influence of every mechanisms quickly, three dimensionless variables $R_{s p} / R_{f}, R_{m} / R_{f}$ and $R_{c} / R_{f}$ are built.

Fig. 4 shows that the perturbation caused by tensile fiber failure descends as soon as the initial velocity increases. Shear plugging is always the main absorbing energy mechanism (within the velocity range considered). At initial velocity below $370 \mathrm{~m} / \mathrm{s}$, the crushing is more important than the momentum transfer. At initial velocity below $100 \mathrm{~m} / \mathrm{s}$, the momentum transfer is almost without influence. As the impact velocity increases, the ratio of $R_{m} / R_{f}$ rises quickly. The reason 
is that the ratio is proportional to square of the projectile velocity. It means that the influence of shear plugging descends as the initial velocity increases, so the solutions between the proposed model and method [19] become closer in Fig. 2.

Fig. 5 shows that the perturbation caused by tensile fiber failure descends as soon as the thickness of laminate increases. Shear plugging is the main absorbing energy mechanism while thickness over $1 \mathrm{~mm}$. Crushing is always more important than momentum transfer. As the impact velocity increases, the ratio of $R_{s p} / R_{f}$ rises quickly. The reason is that the ratio is proportional to the thickness of laminate. It means that the influence of shear plugging rises as the initial velocity increases, so the solutions between the proposed model and method [19] become larger in Fig. 3.

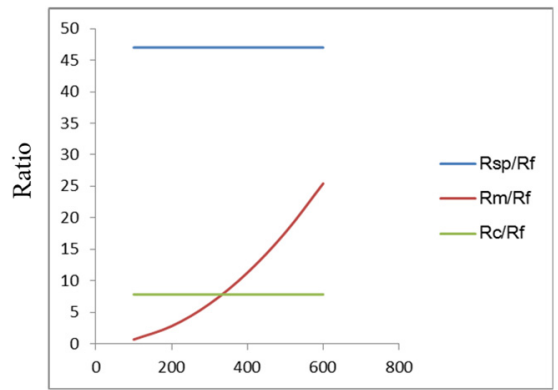

Initial velocity $(\mathrm{m} / \mathrm{s})$

Fig. 4. Ratio of different factor for different projectile initial velocities (thickness $=5 \mathrm{~mm}$ )

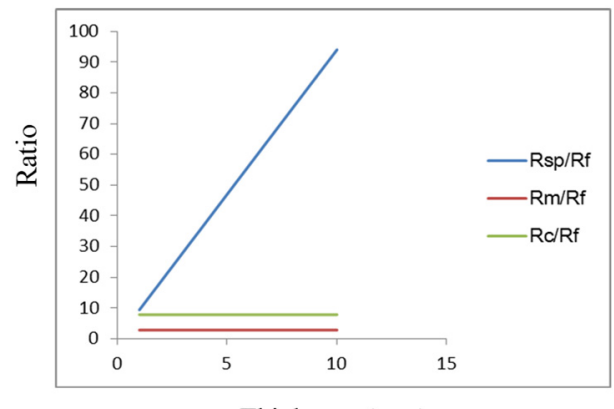

Thickness (mm)

Fig. 5. Ratio of different factor for different laminate thickness $\left(v_{0}=200 \mathrm{~m} / \mathrm{s}\right)$

\section{Conclusions}

An analytical model to predict residual velocity and ballistic limit after high velocity impact on woven Kevlar laminates is proposed. Four different energy absorption mechanisms for the laminate are considered in the model. Then, the model is simplified to obtain the residual velocity and ballistic limit. The analytical result is calculated to compare with that of experiment. The comparison shows a good correlation between the results obtained both numerically and experimental. The perturbation caused by tensile fiber failure descends as soon as the initial velocity or the thickness of laminate increases. The importance of energy absorbing by shear plugging, crushing and the momentum transfer will change by changing of initial velocity of projectile and thickness of laminate. The method of simplification could be useful in preliminary assessment of engineering.

\section{Acknowledgement}

This research is partially supported by the National Natural Science Foundation of China (Grant No. 51475228), the Research Fund of State Key Laboratory of Mechanics and Control of Mechanical Structures (Nanjing University of Aeronautics and Astronautics) (Grant No. MCMS-0517K01) and the Priority Academic Program Development of Jiangsu Higher Education Institutions. The authors wish to thank Mr. Peng Haifeng, Mr. Wushuo and Mr. Li Jin of Chrdi (China) for their technical and financial support. Special thanks also to Mr. Jin Wanzeng and Mr. Mengxiangji for useful discussions.

\section{References}

[1] Bhatnagar A. Lightweight Ballistic Composites Military and Law-Enforcement Applications. Abington Hall, Abington, Cambridge, 2006, p. 364-397. 
[2] Grujicic M., Arakere G. A ballistic material model for cross-plied unidirectional ultra-high molecular-weight polyethylene fibre-reinforced armor-grade composites. Materials Science and Engineering, Vol. 498, 2008, p. 231-241.

[3] Nilakantan G., Keefe M., Gillespie Jr J., Bogetti T. Novel multi-scale modeling of woven fabric composites for use in impact studies. 10th International LSDYNA users conference, Dearborn, Michigan, USA, 2008.

[4] Zhu G., Goldsmith Dharan W. C. H. K. Penetration of laminated Kevlar by projectiles-II, analytical model. International Journal of Solids and Structures, Vol. 29, Issue 4, 1992, p. 421-436.

[5] Sun C. T., Potti S. V. A simple model to predict residual velocity of thick composite laminates subjected to high velocity impact. International Journal of Impact Engineering, Vol. 18, Issue 3, 1996, p. 339-353.

[6] Wen H. M. Predicting the penetration and perforation of FRP laminates struck normally by projectiles with different nose shapes. Composite Structures, Vol. 49, Issue 3, 2000, p. 321-329.

[7] Wen H. M. Penetration and perforation of thick FRP laminates. Composites Science and Technology, Vol. 61, 2001, p. 1163-1172.

[8] Naik N. K., Shrirao P. Composite structures under ballistic impact. Composite Structures, Vol. 66, Issues 1-4, 2004, p. 579-590.

[9] Xuan Haijun, Zhang Na Impact response and damage evolution of triaxial braided carbon/epoxy composites. Part I: Ballistic impact testing. Textile Research Journal, Vol. 83, Issue 16, 2013, p. 1703-1716.

[10] Ganesh Babu M., Velmurugan Gupta R. N. K. Energy absorption and ballistic limit of targets struck by heavy projectile. Latin American Journal of Solids and Structures, Vol. 3, Issue 1, 2006, p. 21-39.

[11] Sikarwar R. S., Velmurugan R., Madhu V. Experimental and analytical study of high velocity impact on Kevlar/Epoxy composite plates. Central European Journal of Engineering, Vol. 2, Issue 4, 2012, p. 638-650.

[12] Sikarwar R. S., Velmurugan R. Ballistic impact on glass/epoxy composite laminates. Defense Science Journal, Vol. 64, Issue 4, 2014, p. 393-399.

[13] Wang Y. B., Wang X. J., Hu X. Z., Wang F. Experimental study of ballistic resistance of Kevlar laminates. Engineering Mechanics, Vol. 22, Issue 3, 2005, p. 76-81.

[14] Wang Y. B., Wang X. J., Bian L., Yu Y. M. CDM model and its application to numerical simulation on fiber-reinforced laminate under penetration. Explosion and Shock Wave, Vol. 28, Issue 2, 2008, p. 172-177.

[15] Yahaya R., Sapuan S. M., Jawaid M., Leman Z., Zainudin E. S. Investigating ballistic impact properties of woven kenaf-aramid hybrid composites. Fibers and Polymers, Vol. 17, Issue 2, 2016, p. 275-281.

[16] Gu Xingjin, Xu Xiwu, Huang Jing High velocity impact damage of thin composite laminates. Journal of Nanjing University of Aeronautics and Astronautics, Vol. 40, Issue 3, 2008, p. 370-375.

[17] Michele Pasquali, Consuelo Terra, Paolo Gaudenzi Analytical modeling of high-velocity impacts on thin woven fabric composite targets. Composite Structure, Vol. 131, 2015, p. 951-965.

[18] Chen Z., Yang G., Zhang C., Wang W. Experimental and modeling study on ballistic impact behavior of a woven carbon/epoxy composite. American Society for Composites Thirty-First Technical Conference, 2016.

[19] Lopez Puente J., Zaera R., Navarro C. An analytical model for high velocity impacts on thin CFRPs woven laminated plates. International Journal of Solids and Structures, Vol. 44, 2007, p. 2837-2851.

[20] Lopez Puente J., Zaera R., Navarro C. Analytical model for high velocity impaction thin CFRPs woven laminated targets. International Journal of Solids and Structures, 2007, p. 1-15.

[21] Naik N. K., Shrirao P., Reddy B. C. K. Ballistic impact behavior of woven fabric composites: formulation. International Journal of Impact Engineering, Vol. 32, 2006, p. 1521-1552.

[22] Naik N. K., Doshi A. V. Ballistic impact behavior of thick composites: parametric studies. Composite Structures, Vol. 82, 2008, p. 447-464.

[23] Brenda Buitrago L., Shirley Garcia Castillo K., Enrique Barbreo Influence of shear plugging in the energy absorbed by thin carbon-fibre laminates subjected to high-velocity impacts. Composites Part B: Engineering, Vol. 49, 2013, p. 86-92. 